\title{
Effectiveness of Combined Therapy of Sertraline and Risperidone in Treating Patient with Obsessive-Compulsive Disorder S Yue ${ }^{1 *}$, H Jiang $^{2 *}$, L Hong $^{3}$, H Deng $^{1}$, MA Ashraf $^{4}$
}

\begin{abstract}
Objective: To research the effectiveness of combined therapy of sertraline and risperidone in treating patient with obsessive-compulsive disorder (OCD).

Method: By analyzing the pathological mechanism of OCD, this paper employed combined therapy of sertraline and risperidone to treat OCD patient, and explored the clinical effect of the combined therapy.
\end{abstract}

Results: After combined therapy treatment, OCD symptoms were significantly improved. Compared with traditional therapies, the combined therapy is of more significant effect. Common adverse reactions of drug therapy mainly include intestinal discomfort, headache, insomnia, lack of strength and anxiety.

Conclusion: The combined therapy of sertraline and risperidone has advantages including sound efficacy, instant effect, high safety, and insignificant adverse reaction, which should be widely promoted.

Keywords: OCD Patient, Sertraline, Risperidone, Therapy

*Authors Contribution: The first two authors (Suping Yue and Hanwen Jiang) contributed equally to this work.

From: Center, West China Hospital of Sichuan University, Chengdu, 610041, ${ }^{2}$ Emergency Department, The Second Affiliated Hospital of Chengdu Medical College, Chengdu, 610000, ${ }^{3}$ Psychiatry Department, Capital Medical University affiliated Beijing Anding Hospital, Beijing, 100000, China, ${ }^{4}$ Faculty of Science and Natural Resources, University Malaysia Sabah 88400 Kota Kinabalu, Sabah, Malaysia.

Correspondence: Dr H Deng, Mental Health Center, West China Hospital of Sichuan University, Chengdu, 610041,China. Email: rhdeng88@ hotmail.com 


\section{INTRODUCTION}

In clinical practice, $\mathrm{OCD}$ is kind of neurosis represented by symptoms including obsessive-compulsive ideas and behaviors. OCD brings different degrees of suffers and anxieties, which severely impact patient's life quality. OCD occurs among people in different ages from child to elderly people. The elderly people, in particular, most of which have suffered physical diseases already, OCD brings mental problems to them. The OCD symptoms of elderly patients are dominated by OC ideas, which lead to bad psychologies such as pain, desperation, helplessness. In recent year, the combined therapy of sertraline and risperidone has achieved significant therapeutic effect in clinical practice. The combined therapy can increase clinical efficacy and prognosis with higher safety; however difference may occur when using one of the drugs solely (1). To further research the clinical effect and safety in treatment of OCD, the combined therapy sertraline and risperidone was employed as the major treatment, and clinical reports are shown below.

\section{SUBJECT AND METHOD}

\section{Pathological mechanism of OCD}

OCD pathology: Currently the cause for OCD is not very clear, however there have been large numbers of researches showing that OCD is related to anxiety disorder, genetic factors, personality, adverse event, stimulation factors and so on, of which, the personality is the most relative factor. OCD symptoms: 1 . Obsessive ideas including suspicion, recall, rumination, etc; 2. Obsessive behaviors such as repeatedly checking, repeatedly washing hands, 
repeatedly counting, compulsive rituals, etc. Of all factors, the most dominate cause is the psychological factor.

According to related investigation results, in Shanghai there are 38\% OCD patients who have mental disorders before OCD attacking. Those psychological factors which can lead to long-term consideration, anxiety and tension and those emergency events which bring serious shock are called induced factor of OCD. During OCD pathogenic process, social psychological factors can never be ignored. Being in state of physical fatigue for long is easy to induce OCD for patients who have compulsive character. On the other hand, there are different explanations for the pathogenesis of OCD. According to scholar of Pavlov school, in experiencing intensive emotions, human brain is subjected to cortex excitability, hypertension and psychological conflict in excitoinhibitory process, the pathological inert focus of excitation will be constructed, which thus leads to the formation of obsessive ideas and eventually the occurrence of OCD. However based on the research of psychological school, OCD is mainly induced by the restraining of aggressive impulse; Even some people who proposed their opinion through theoretical explanation that obsessive idea is the result of anxiety and simulation as well as the conditioned connection between ideas. The Basilar artery is shown as Figure 1. The Comparison of OCD and anxiety disorder nerve plate is shown as Figure 2. The two-sample brain activation map is shown as Figure 3.

\section{Research design}

OCD patients treated by in our hospital in 2015 are selected as research objects in a randomized controlled trial. All qualified OCD patients are divided into control group and treatment group, wherein the former group is treated with sertraline while treatment group is 
treated with combined therapy of sertraline and risperidone. Research design and data collection processes shown in Figure 4.

\section{Therapeutic method}

During treating process, the control group is treated with sertraline, of which the initial dosage is controlled at $50 \sim 100 \mathrm{mg} / \mathrm{d}$. With the development of illness, the dosage can be increased and controlled at $50 \mathrm{mg} / \mathrm{d}$ after two weeks of medication, wherein the largest dosage is approximately $200 \mathrm{mg} / \mathrm{d}$; On the basis of therapy of control group, the treatment group is treated by additionally adding risperidone, of which the initial dosage is risperidone, largest dosage is controlled at $4 \mathrm{mg} / \mathrm{d}$. Before treatment of both groups, other types of antipsychotic drugs are prohibited to be taken (2). The Comparison of ALEF between OCD patient and normal people under resting-state is shown as Figure 5.

\section{RESULTS}

\section{Basic data}

The distribution of demographics data of all selected OCD patients are: average age is 44.65 \pm 7.5 , wherein the youngest is 18 years old, while the oldest is 80 years old. Patients under 80 years account for $36.21 \%$, while patients above 18 years account for $63.79 \%$, and the elderly patients are relatively more; Female patients are slightly more than male patients; Patients at educational level of illiteracy, primary school, junior high account for $62.93 \%$, patients at level of senior high account for 37.07\%; Most patients are in marriage, accounting for 93.10\%; Except one patient who is religious, other patients are not religious; Majority of 
patients live with spouses, son or daughter, grandson or granddaughter, accounting for 94.83\%; Most patients are now in non-duty status, accounting for $80.21 \%$; Most patients can take care of themselves, account for $86.21 \%$; Most patients are of economic self-sufficiency, accounting for $66.38 \%$, while $33.62 \%$ live by partial or whole living subsidies from government and their son or daughter; Most patients pay medical expense by medical insurance, accounting for $71.55 \%$.

\section{Therapeutic effect}

According to Y-BOCS score-reducing rate: Recovery $\geq 75 \%$, significantly effective $\geq 50 \%$, effective $\geq 25 \%$, Non-effective $<25 \%$. In treatment group, there are 300 patients with recovery, 150 patients with significantly effect, 50 patients with effect, 0 patient with non-effect, achieving total effective rate of $100 \%$; while in control group, there are 210 patients with recovery, 90 with significant effect, 120 with effect, 80 with non-effect, achieving total effective rate of $89.76 \%$; By comparing both groups, it can find that treatment group achieve more significant therapeutic effect. The therapeutic effect of both groups after treatment is shown as Table 1. The comparison of Y-BOCS, HAMA, HAMD scores before and after treatment is shown as Table 2.

\section{Adverse reactions}

After treatment, there are totally 4 patients suffering adverse reactions, wherein 3 patients in control group show adverse reactions including gastrointestinal discomfort (1), headache and insomnia (1), lack of strength and anxiety (1), concluding the occurrence rate of adverse reaction with $25.65 \%$; while in treatment group, there is only one showing adverse reaction of gastrointestinal discomfort (including nausea, vomiting, anorexia, diarrhea, etc), and the 
occurrence rate of adverse reaction is $8.76 \%$; Through comparing two groups, it can be seen that the treatment is significantly lower that control group in terms of occurrence rate of adverse reaction, and the differences between both groups are of statistical significance $(\mathrm{P}<0.05)$. The correlation analysis of Y-BOCS score and ALEF value of OCD patient is shown as Figure 6.

\section{DISCUSSION}

In recent years, with the progress of China's national economy, people are suffering an increasingly economic stress and mental stress, therefore the number of OCD patients' increases dramatically. Bringing various safety risks for normal daily life and social stability, this disease must be laid with priority of prevention and treatment (3). Beside high complex pathological mechanism, OCD is also a disease with extremely high recurrence rate. The inducing factor is related to abnormal serotonin value and decreased overall activity of dopamine. Once being affected by OCD, patient's limbic system is in hyperfunctional state, which will directly impact normal daily life and life health (3).

As a common drug for treating OCD, risperidone is a benzisoxazole derivative, which has strong affinity with D2 receptor and 5-HT2 receptor. After applying on D2 receptor, it can effectively relieve positive symptoms (6). Moreover, 5-HT2 receptor can further relieve patient's emotional symptoms and negative symptoms. By interdicting the excitation ability of norepinephrine and 5-hydroxytryptamine (5-HT2A) receptor, it can relieve negative symptoms.

Sertraline is common drug for OCD and usualy made in pills. Being able to reuptake 
5-hydroxytryptamine and to selectively inhibit central nervous system, sertraline can significantly increase the content of 5-hydroxytryptamine in synaptic cleft. Therefore, it is of certain anti-depression effect and can be used for OCD treatment (7). Compared with other drugs, sertraline can not only reduce depressive symptoms, but also reduce fidgety mood, long-term fatigue and anxiety. In addition, in aspect of OCD treatment, sertraline is more significantly effective with good tolerance and high safety, and can serve as one of priority drugs for OCD.

Currently the cause for OCD is not very clear, however there have been large numbers of researches showing that OCD is related to anxiety disorder, genetic factors, personality, adverse event, stimulation factors and so on, of which, the personality is the most relative factor. Therefore, large amounts of researches have showed that the combined therapy of sertraline and risperidone has achieved significant efficacy in clinical practice (5). The OCD patients treated by in our hospital in 2015 are selected as research objects in a randomized controlled trial. All qualified OCD patients are divided into control group and treatment group, wherein the former group is treated with sertraline while treatment group is treated with combined therapy of sertraline and risperidone.

According to Y-BOCS score-reducing rate: Recovery $\geq 75 \%$, significantly effective $\geq 50 \%$, effective $\geq 25 \%$, Non-effective $<25 \%$. Treatment group achieves total effective rate of $100 \%$; while the control group achieve total effective rate of $89.76 \%$. By comparing both groups, it can find that treatment group achieve more significant therapeutic effect $(\mathrm{P}<0.05)$. After treatment, there are totally 4 patients suffering adverse reactions, wherein 3 patients in control group show adverse reactions including gastrointestinal discomfort (1), headache and 
insomnia (1), lack of strength and anxiety (1), concluding the occurrence rate of adverse reaction with $25.65 \%$; while in treatment group, there is only one showing adverse reaction of gastrointestinal discomfort (including nausea, vomiting, anorexia, diarrhea, etc), and the occurrence rate of adverse reaction is $8.76 \%$. Through comparing two groups, it can be seen that the treatment is significantly lower that control group in terms of occurrence rate of adverse reaction, and the differences between both groups are of statistical significance $(\mathrm{P}<$ $0.05)$.

\section{CONCLUSION}

In all, the combined therapy of sertraline and risperidone can be used to treat refractory OCD with advantages including sound efficacy, instant effect, high safety, and insignificant adverse reaction, which should be widely promoted.

\section{ACKNOWLEDGEMENT}

Authors Contribution: The first two authors (Suping Yue and Hanwen Jiang) contributed equally to this work. 


\section{REFERENCES}

1. Wang B, Lu Q. Randomized controlled rrial of combined therapy of sertraline and risperidone in treating OCD. J Contemporary Medicine 2010; 28: 88-9.

2. Liu J, Li Z, Li Yi. Controlled study of combined therapy of sertraline and paroxetine and risperidone in treating OCD with psychotic symptoms. J Medical Forum 2011; 14: 133-5.

3. Zhao Y, Zhang Y, Yu X. Efficacy of combined therapy of risperidone and sertraline in treating OCD. J China J Health Psychology 2011; 10: 1196-7.

4. Wang F, Li H. Observation of efficacy of combined therapy of sertraline and risperidone in treating schizophrenic OCD. J Chinese J Pharmacoepidemiology 2015; 6: $338-40$.

5. Ma R, Ma Y, Li Y. 43 Cases study-efficacy of combined therapy of sertraline and risperidone in treating elderly OCD. J China Pharmaceutical 2015; 15: 100-1.

6. Liu J, Li Z, Li Y. Clinical research of combined therapy of sertraline and small dosage of risperidone in treating refractory OCD. J Chinese J Practical Nervous Diseases 2012; 8: 24-6.

7. Liu Y, Zhang H, Liu K, Liang X. Randomized controlled trial of combined therapy of fluvoxamine and risperidone in treating children's OCD. J China J Health Psychology 2012; 10: 1460-2. 
Table 1: Therapeutic effects of both groups after treatment [n (\%)]

\begin{tabular}{lllllll}
\hline Group & $\begin{array}{l}\text { No of } \\
\text { patient }\end{array}$ & Recovery & $\begin{array}{l}\text { Significant } \\
\text { ly effect }\end{array}$ & Effective & $\begin{array}{c}\text { Non-effect } \\
\text { rate (\%) }\end{array}$ \\
\hline $\begin{array}{l}\text { Treatment } \\
\text { group }\end{array}$ & 500 & 300 & 150 & 50 & 0 & 100 \\
$\begin{array}{l}\text { Control } \\
\text { group }\end{array}$ & 500 & 210 & 90 & 120 & 80 & 89.76 \\
\hline
\end{tabular}

Table 2: Comparison of Y-BOCS, HAMA, HAMD scores before and after treatment $(\mathrm{n}=20, \bar{x} \pm \mathrm{s})$

\begin{tabular}{llllll}
\hline Group & $\begin{array}{l}\text { No of } \\
\text { patient }\end{array}$ & $\begin{array}{l}\text { Y-BOCS } \\
\text { Before and } \\
\text { after treatment }\end{array}$ & $\begin{array}{l}\text { Y-BOCS } \\
\text { Before and after } \\
\text { treatment }\end{array}$ & $\begin{array}{l}\text { Y-BOCS } \\
\text { Before and after } \\
\text { treatment }\end{array}$ \\
\hline Treatment & 50 & $21.43 \pm 3.43$ & $12.76 \pm 6.76$ & $11.67 \pm 5.23$ & $4.77 \pm 2.11$ \\
group & 0 & $8.94 \pm 3.21$ & $4.65 \pm 1.46$ & & \\
Control & 50 & $20.32 \pm 5.22$ & $13.65 \pm 5.76$ & $11.78 \pm 6.87$ & $7.90 \pm 3.87$ \\
group & 0 & $11.54 \pm 2.43$ & $8.65 \pm 2.67$ & & \\
\hline
\end{tabular}




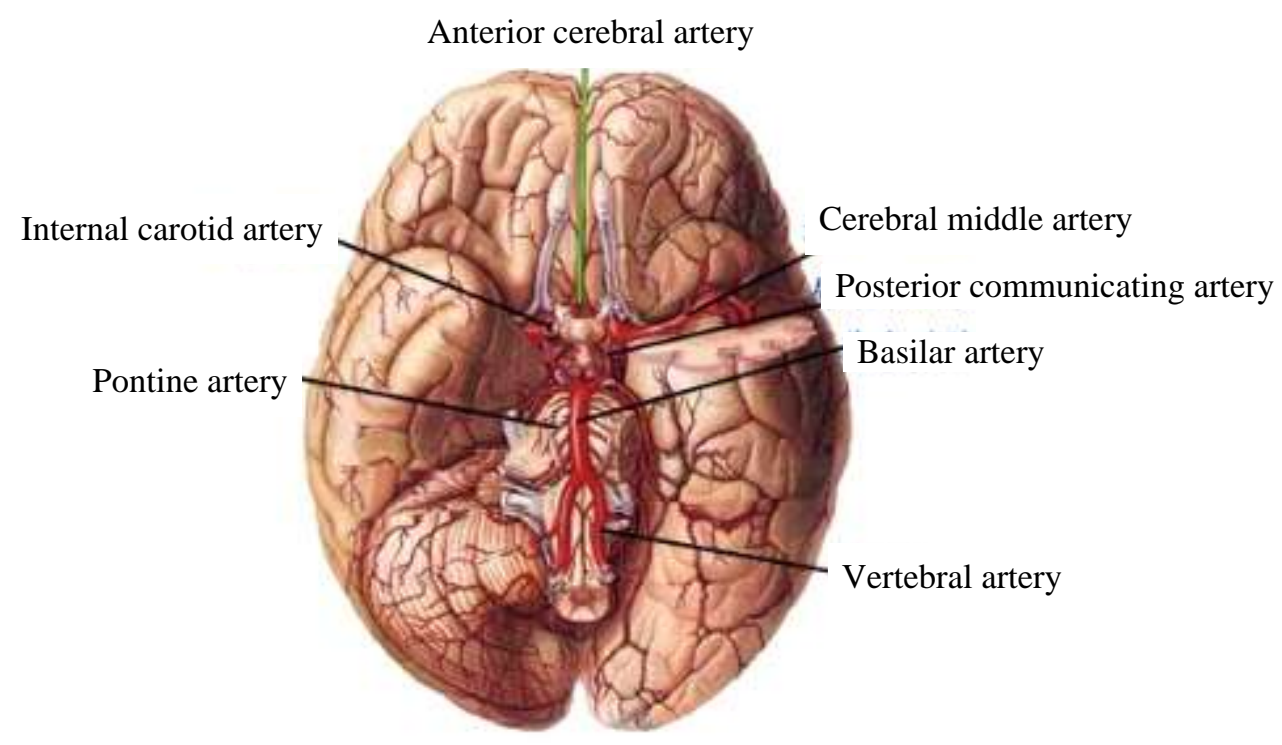

Fig. 1: Basilar artery.
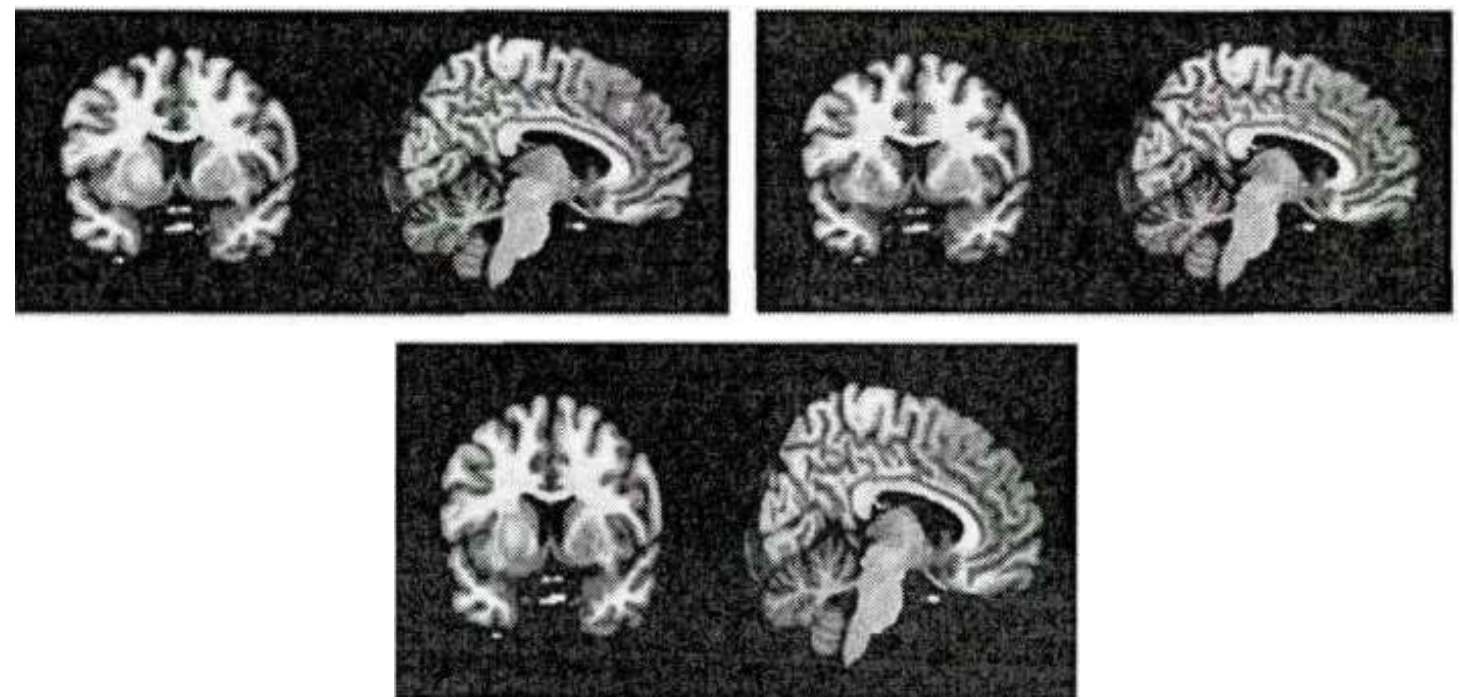

Fig. 2: Comparison of OCD and anxiety disorder nerve plate 

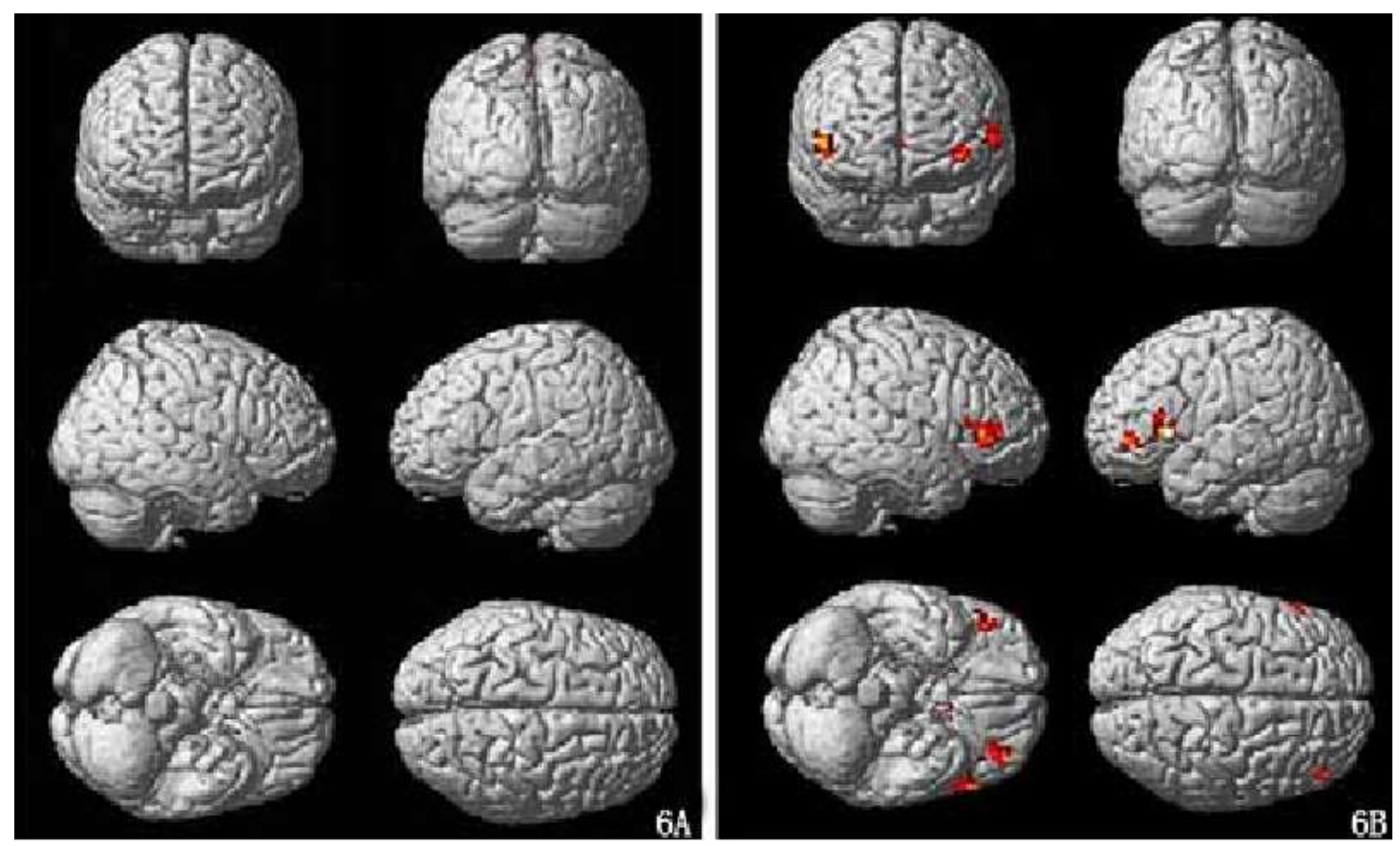

Fig. 3: Two-sample brain activation map.

6A: Activated encephalic region of OCD patient is not higher than that of normal control group 6B: Activated encephalic region of OCD patient is lower than that of normal control group mainly including left anterior cingutate, nucleus caudatus and bilateral orbitofrontal cortices 


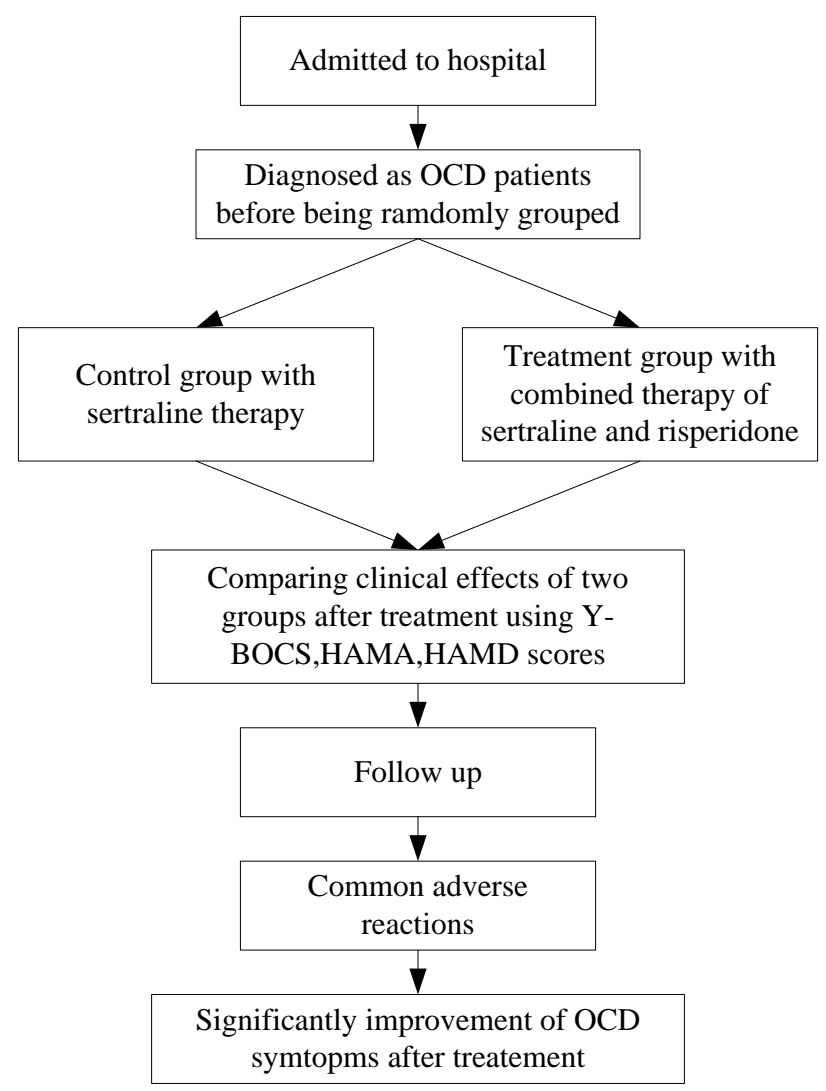

Fig. 4: Flow chart of data collection.

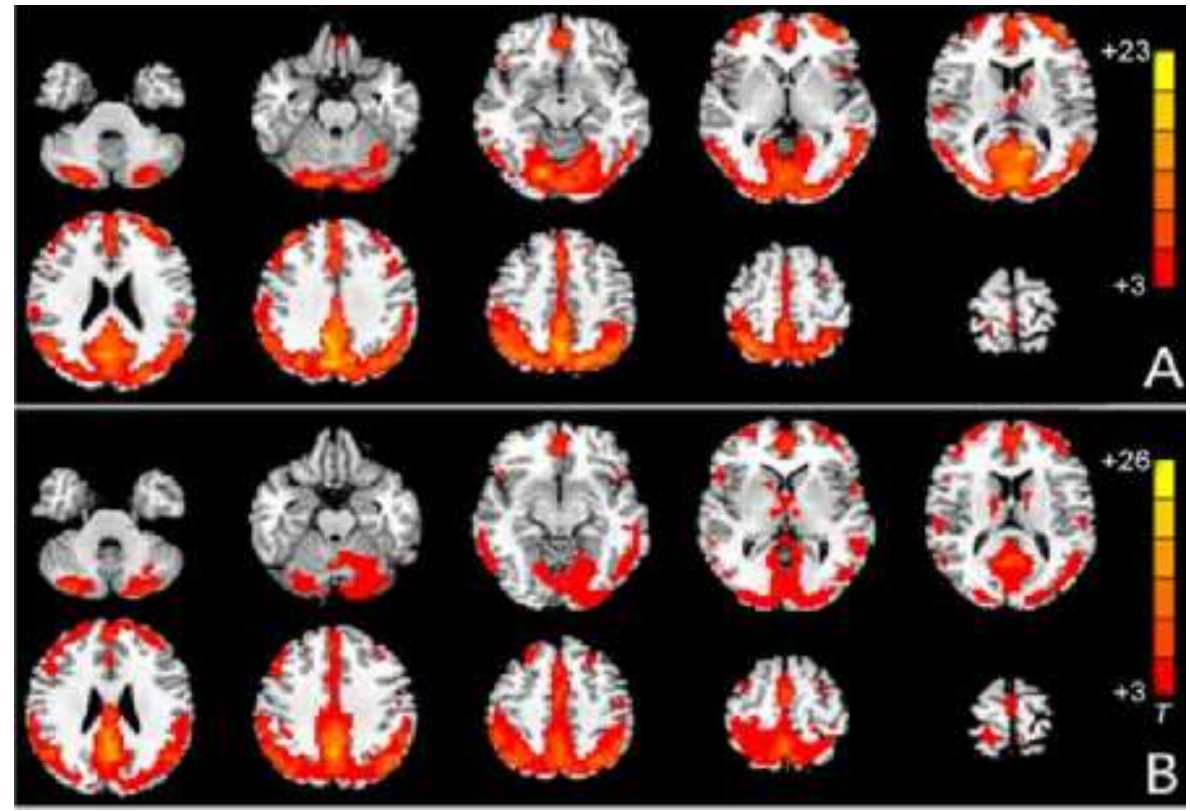

Fig. 5: Comparison of ALEF between OCD patient and normal people under resting-state A: ALEF of OCD patient; $B$ : ALEF of normal people ( $\mathrm{P}<0.01$, corrected by Alphasim) 

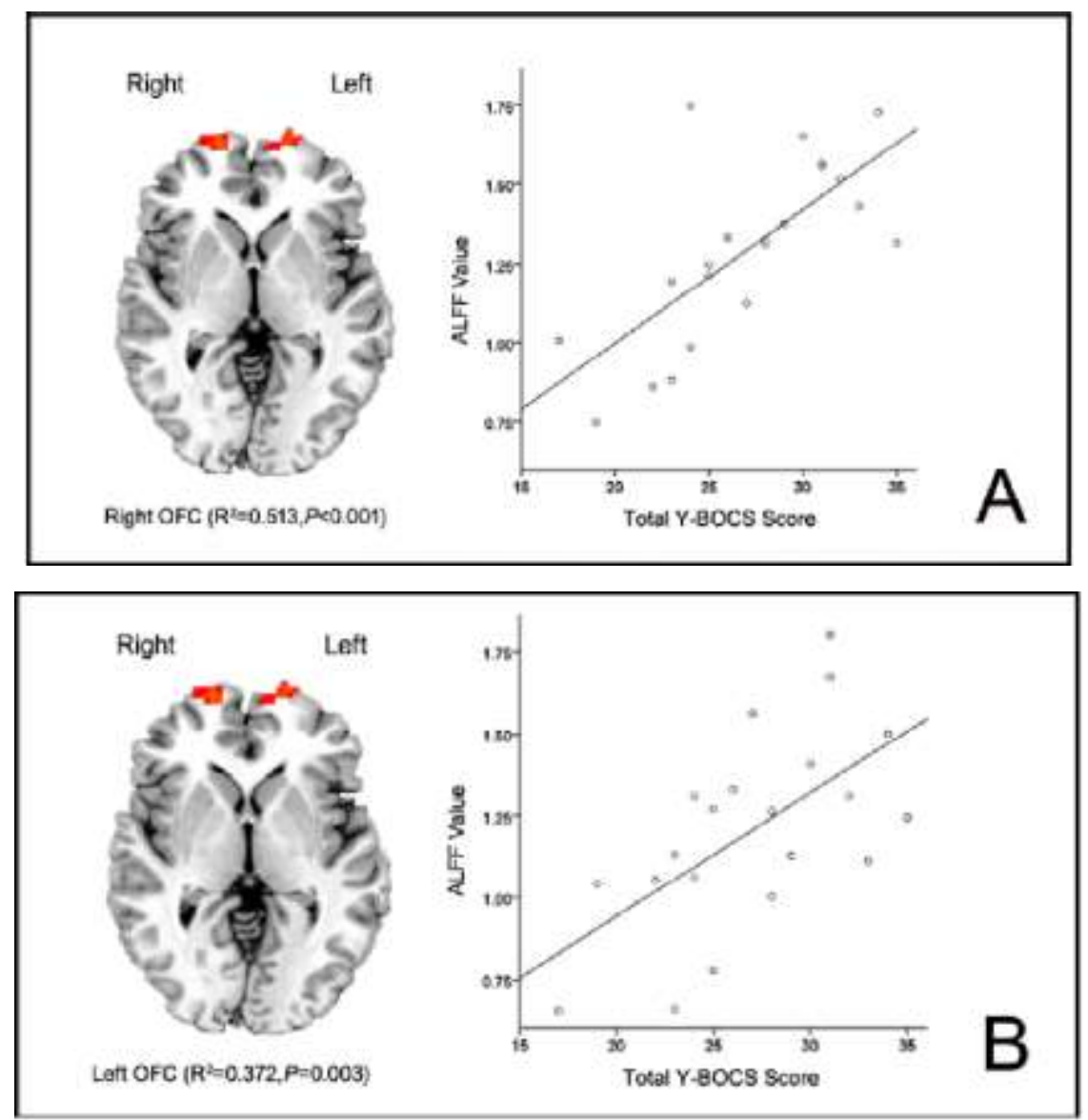

Fig. 6: Correlation analysis of Y-BOCS score and ALEF value of OCD patient

A Result of correlation analysis of right orbitofrontal cortex ; B Result of correlation analysis of left orbitofrontal cortex (non-corrected, $\mathrm{P}<0.005$ ). 